\title{
Modulation Effects of Cordycepin on Voltage-Gated Sodium Channels in Rat Hippocampal CA1 Pyramidal Neurons in the Presence/Absence of Oxygen
}

\author{
Zhi-Bin Liu, ${ }^{1}$ Chao Liu, ${ }^{2,3}$ Bin Zeng, ${ }^{2}$ Li-Ping Huang, ${ }^{3}$ and Li-Hua Yao ${ }^{1,2}$ \\ ${ }^{1}$ School of Sport Science, Jiangxi Science \& Technology Normal University, Nanchang, Jiangxi 330013, China \\ ${ }^{2}$ School of Life Science, Jiangxi Science \& Technology Normal University, Nanchang, Jiangxi 330013, China \\ ${ }^{3}$ School of Pharmacy, Jiangxi University of Traditional Chinese Medicine, Nanchang, Jiangxi 330004, China \\ Correspondence should be addressed to Li-Hua Yao; yaolh79@yahoo.com
}

Received 6 July 2017; Accepted 4 October 2017; Published 31 October 2017

Academic Editor: Clive R. Bramham

Copyright (c) 2017 Zhi-Bin Liu et al. This is an open access article distributed under the Creative Commons Attribution License, which permits unrestricted use, distribution, and reproduction in any medium, provided the original work is properly cited.

\begin{abstract}
Our previous study revealed that cordycepin features important neuroprotective effects against hypoxic insult by improvement of neuronal electrophysiological function. Modulation on voltage-gated sodium channel (VGSC) in CA1 neurons is the initial event during hypoxia/ischemia. However, no study comprehensively investigated cordycepin on VGSC. Hence, this study investigated modulation effects of cordycepin on VGSC not only in oxygen physiological conditions but also in acute oxygen deprivation injury conditions. Results revealed that cordycepin $(80 \mu \mathrm{M})$ reduced the amplitude of VGSC currents $\left(I_{\mathrm{Na}}\right)(77.6 \%$ of control, $p<0.01)$ within 1 min of drug exposure coupled with a negative shift in steady-state inactivation and prolonged recovery time course from inactivation. Additionally, this mild reduction on the peak of $I_{\mathrm{Na}}$ induced by the pretreatment with cordycepin can attenuate and delay the following hypoxia causing rapid dramatic decrease in $I_{\mathrm{Na}}$ with no additive change in the voltage dependence of inactivation. As modulation on VGSC in CA1 neurons represents the initial event during ischemia, we propose that suppression effect of cordycepin on VGSC is an important neuronal protective mechanism that may enhance neuronal tolerance to acute oxygen deprivation and delay hypoxia-induced neuronal injuries.
\end{abstract}

\section{Introduction}

Voltage-gated sodium channel (VGSC) plays a significant role in neuronal functions of the central nervous system, which is responsible for initiation and propagation of the neuronal action potential. Thus, VGSC is critical in signal communication between neurons and participates in regulating various physiological functions $[1,2]$. In addition to its involvement in normal physiological events, increasing evidence suggests that VGSC also plays a key role in regulating pathophysiological processes, such as hypoxia [1-5]. Inhibition of VGSC activation results in reduced neuronal activity and $\mathrm{Na}^{+}$influx across neuronal membrane, which in turn reduces metabolic demand on neurons in cases when energy production is severely compromised $[2,3]$. This process ultimately increases neuronal tolerance to low-oxygen environments. Thus, inhibition of $I_{\mathrm{Na}}$ is usually considered as a cellular protective mechanism during initial stages of hypoxia [2-5].

Therefore, compounds modulating VGSC are developed for neuroprotective treatments $[2,4,5]$. Recent reports from our laboratory focused on the development of novel pharmacological actions from traditional Chinese medicine natural products. Cordycepin (3-deoxyadenosine), a major functional component of Cordyceps militaris [6], exhibits a wide range of biological effects, including antitumor [7, 8], anti-inflammatory $[9,10]$, antidiabetic $[11,12]$, and antioxidant activities $[13,14]$. Recent reports suggested that cordycepin features neuroprotective effects on neuronal damage caused by ischemia/reperfusion insult by reducing oxidative damage, increasing free radical scavenging activity, and preventing neuronal cell death [13-15].

Studies from our laboratory demonstrated that cordycepin can increase neuronal tolerance during hypoxia and 
delay hypoxia-induced membrane depolarization and that the mechanism to suppress the neuron activity is strongly involved [16, 17]. As inhibition of VGSC activation would result in reduced neuronal activity and $\mathrm{Na}^{+}$influx across the neuronal membrane, activation of VGSC plays a critical role in mediating sustained $\mathrm{Na}^{+}$entry during ischemia and hypoxia, which then induce membrane depolarization $[3,18]$. Thus, blocking these channels may exert neuroprotection during hypoxia [4,5]. Although our previous study discovered that cordycepin selectively regulates activities of whole-cell $\mathrm{Na}^{+}$current $\left(I_{\mathrm{Na}}\right)$, no study comprehensively investigated its regulating mechanism [19]. Hence, in this study, the effect of cordycepin on the kinetics of VGSC in the hippocampal CA1 pyramidal neurons was investigated by using whole-cell patch-clamp techniques under voltageclamp configuration [2]. Finally, actions of cordycepin on VGSC were also evaluated under hypoxia by using an energy-deprived injury model $[16,20]$.

\section{Material and Methods}

2.1. Drug Preparation. Chemicals used for making artificial cerebrospinal fluid (ACSF), tetrodotoxin (TTX), tetraethylammonium chloride (TEA-Cl), 4-aminopyridine (4-AP), $\mathrm{Na}_{2}-\mathrm{ATP}, \mathrm{CsCl}_{2}$, ethylene glycol tetraacetic acid (EGTA), $\mathrm{CdCl}_{2}$, and hydroxyethyl piperazineethanesulfonic acid (HEPES) were purchased from Sigma Co. (St. Louis, MO, USA). Cordycepin with $98 \%$ purity was provided by South China Normal University [16, 21].

2.2. Preparation of Hippocampal Brain Slices. Animal studies were approved by the Institutional Care and Use Committee of Jiangxi Science and Technology Normal University. All experiments were performed on CA1 pyramidal neurons of hippocampal brain slices prepared from 15 to 22-day-old Sprague-Dawley rats as described in our previous studies $[16,22]$. Animals were anesthetized with isoflurane and decapitated. The brains were quickly removed from the cranial cavity and immersed in ice-cold $\left(4^{\circ} \mathrm{C}\right)$ oxygenated (95\% $\mathrm{O}_{2} / 5 \% \mathrm{CO}_{2}$ ) ACSF containing the following (in $\mathrm{mM}$ ): $\mathrm{NaCl} 117, \mathrm{KCl} 4.7, \mathrm{MgCl}_{2} 1.2, \mathrm{NaH}_{2} \mathrm{PO}_{4}$ 1.2, $\mathrm{NaHCO}_{3} 25$, $\mathrm{CaCl}_{2} 2.5$, and D-glucose 10 (pH 7.4). Osmolarity of bathing solution was adjusted to 325-330 mOsm with sucrose. Hippocampus was dissected free, and transverse hippocampal slices ( $400 \mu \mathrm{m}$ in thickness) were obtained using a vibrating microtome (NVSLM1, World Precision Instruments, USA). Slices were allowed to recover in continuously oxygenated ACSF for at least $1 \mathrm{~h}$ prior to experiments.

2.3. Patch-Clamp Recording. After recovery, individual slices were transferred to a recording chamber, which was continually perfused with oxygenated ACSF (unless otherwise indicated) at a rate of $4 \mathrm{ml} / \mathrm{min}$. All experiments were performed at room temperature $\left(26^{\circ} \mathrm{C}\right)$. Hypoxia was induced by switching from oxygenated ACSF equilibrated with $95 \% \mathrm{O}_{2} / 5 \% \mathrm{CO}_{2}$ to the same bath solution equilibrated with $95 \% \mathrm{~N}_{2} / 5 \% \mathrm{CO}_{2}$ for $>2 \mathrm{~h}$. Bath solution was exchanged within $\sim 20 \mathrm{~s}$.
The following experiments were performed using conventional whole-cell patch recording under voltage-clamp configuration. Currents through VGSC were measured using a MultiClamp 700B patch-clamp amplifier (Axon Instruments, USA). Recording electrodes were fabricated from borosilicate glass pipettes (Sutter Instruments, USA) by a Flaming-Brown puller (P-97, Sutter Instruments, USA) and were filled with intracellular solution containing (in $\mathrm{mM}$ ) $\mathrm{CsCl}_{2}$ 140, TEA-Cl 5, ATP-Na 2 2, EGTA 10, and HEPES 10 ( $\mathrm{pH} 7.2$ ) [19]. Electrode resistance reached 4-6 M $\Omega$ when pipettes were filled with solution.

Bathing solution was supplemented with $25 \mathrm{mM}$ TEA$\mathrm{Cl}, 5 \mathrm{mM} 4-\mathrm{AP}$, and $0.4 \mathrm{mM} \mathrm{CdCl}_{2}$ to block delayed rectified $\mathrm{K}^{+}$channels, transient outward $\mathrm{K}^{+}$channels, and all $\mathrm{Ca}^{2+}$ channels, respectively [2, 19]. Recorded neuronal cells were allowed to stabilize for 1-2 min till the stable conditions. Cordycepin was dissolved in ACSF at concentrations of $20,40,80$, and $200 \mu \mathrm{M}$, and its effects were tested by bath perfusion (solution exchange was completed in about $20 \mathrm{~s}$ ).

2.4. Data Analysis. Data were acquired by Clampex 10.5 via a digidata 1322 series A/D (Axon Instruments, USA) board at a sampling frequency of $20 \mathrm{kHz}$. Series resistance (10-20 M $\Omega$ ) was monitored during the recording, and cells with changes $>30 \%$ in the series resistance were abandoned. Only one CA1 pyramidal neuron was tested in a hippocampal slice after the successful recording was made. Therefore, the number of samples $(n)$ in each test group represents the cells recorded from different hippocampal slices.

Electrophysiological parameters were measured as previously described $[2,19]$. For current-voltage activation curve plot, the current amplitudes before and after the cordycepin application were all expressed as percentages of the maximum current amplitude recorded initially under control conditions. For inactivation plots, the currents were normalized to their corresponding maximum values before and after cordycepin application. For recovery from inactivation plots, the recovery current amplitude (normalized with respect to the precondition pulse induced the current amplitude) was plotted versus times before and after cordycepin application. Steady-state activation kinetics of $I_{\mathrm{Na}}$ were obtained using a Boltzmann fit equation $G / G_{\max }=1 /\left(1+\exp \left(\left(V_{1 / 2}-V_{\mathrm{m}}\right) /\right.\right.$ $\left.V_{\mathrm{c}}\right)$ ), where $G$ represents conductance at each command voltage, $G_{\max }$ refers to maximal conductance, $V_{\mathrm{m}}$ corresponds to command voltage, $V_{1 / 2}$ denotes half-maximal activation, and $V_{\mathrm{c}}$ is proportional to the slope at $V_{1 / 2}$. Steady-state inactivation of $I_{\mathrm{Na}}$ was obtained with another Boltzmann fit equation $I / I_{\max }=1 /\left(1+\exp \left(\left(V_{\mathrm{m}}-V_{1 / 2}\right) / V_{\mathrm{c}}\right)\right)$, where $I_{\max }$ refers to maximal current amplitude, $I$ represents the current amplitude measured from each command voltage, $V_{\mathrm{m}}$ corresponds to conditioning voltage, $V_{1 / 2}$ denotes half-maximal inactivation, and $V_{\mathrm{c}}$ is proportional to the slope at $V_{1 / 2}$. The time-to-peak value was used to analyze activation kinetics. Inactivation and deactivation time constants were obtained by fitting the current traces monoexponentially.

Results were presented as mean \pm SEM. Statistical significance of difference was calculated using two-tailed 

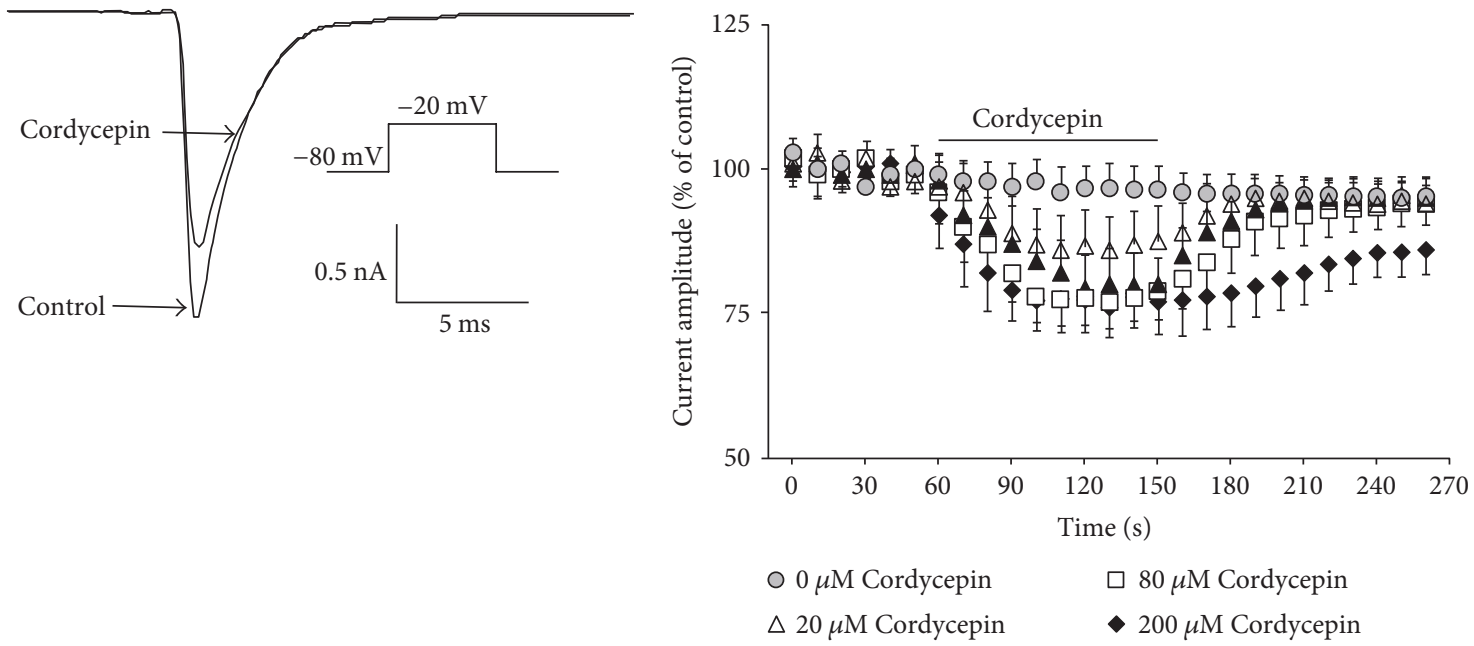

(a)

(b)

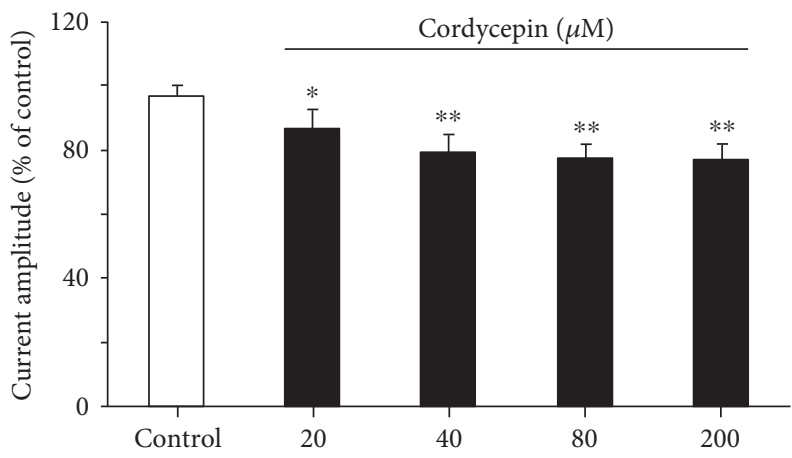

(c)

FIGURE 1: Cordycepin suppressed the amplitude of $I_{\mathrm{Na}}$ in a concentration-dependent manner in rat hippocampal CA1 pyramidal neurons. (a) Representative traces of evoked $I_{\mathrm{Na}}$ before and after $80 \mu \mathrm{M}$ cordycepin application in a whole-cell patch-clamp configuration. (b) Time courses of the effects of different concentrations of cordycepin $(0,20,40,80$, and $200 \mu \mathrm{M})$ on $I_{\mathrm{Na}}$ amplitude. Note that the bath application of cordycepin is indicated by a horizontal bar. (b) Effects of different concentrations of cordycepin $(20,40,80$, and $200 \mu \mathrm{M})$ on $I_{\mathrm{Na}}$. The amplitude of $I_{\mathrm{Na}}$ after cordycepin application was expressed as a percentage of $I_{\mathrm{Na}}$ amplitude before cordycepin application. ${ }^{*} p<0.05$, ${ }^{* *} p<0.01$ as compared with the control group.

Student's $t$-test. $p<0.05$ level of confidence was considered statistically significant.

\section{Results}

3.1. Cordycepin Inhibited $I_{N a}$ in a Concentration-Dependent Manner. $I_{\mathrm{Na}}$ was activated by using a step depolarization test pulse with a $50 \mathrm{~ms}$ duration from a holding membrane potential of $-80 \mathrm{mV}$ to $-20 \mathrm{mV}$. In these experiments, $I_{\mathrm{Na}}$ was recorded at $10 \mathrm{~s}$ intervals. When $I_{\mathrm{Na}}$ reached a stable maximum amplitude, cordycepin was applied by bath perfusion. As shown in Figure 1, the amplitude of $I_{\mathrm{Na}}$ decreased in the presence of $80 \mu \mathrm{M}$ cordycepin $(77.6 \% \pm 4.58 \%$ of the control, $n=10 ; p<0.01)$. After cordycepin $(80 \mu \mathrm{M})$ reached the chamber, inhibitory effects on $I_{\mathrm{Na}}$ occurred immediately and reached the maximum and stable value within $1 \mathrm{~min}$, coinciding with our previous study [19]. Inhibition of cordycepin on the $I_{\mathrm{Na}}$ was concentration dependent (Figures 1(b) and $1(\mathrm{c}))$. As concentration of $80 \mu \mathrm{M}$ cordycepin caused maximal effects and can be washed out quickly (Figures 1(b) and 1(c)), this concentration was adopted for subsequent tests.

3.2. Effects of Cordycepin on $I_{\mathrm{Na}}$ Steady-State Activation. From a holding potential of $-80 \mathrm{mV}$, active currents were evoked by a series of $+10 \mathrm{mV}$ voltage steps to potential with $50 \mathrm{~ms}$ duration between -80 and $+40 \mathrm{mV}$ to test the effect of cordycepin on $I_{\mathrm{Na}}$ steady-state activation. Figure 2(a) shows representative raw traces from both control and $80 \mu \mathrm{M}$ cordycepin recordings.

As shown in Figure 2(b), threshold for activation of $I_{\mathrm{Na}}$ measured $-60 \mathrm{mV}$, and the amplitude of $I_{\mathrm{Na}}$ was maximal at $-20 \mathrm{mV}$ in the control and cordycepin-treated groups. The amplitude after cordycepin treatment was significantly lower than that of control at most voltage points (Figure 2(b)). However, steady-state activation curves for $I_{\mathrm{Na}}$ in the control $(n=10)$ and cordycepin $(n=10)$ treatment groups did not show a significant shift, as shown in Figure 2(c) $(p>0.05)$. 

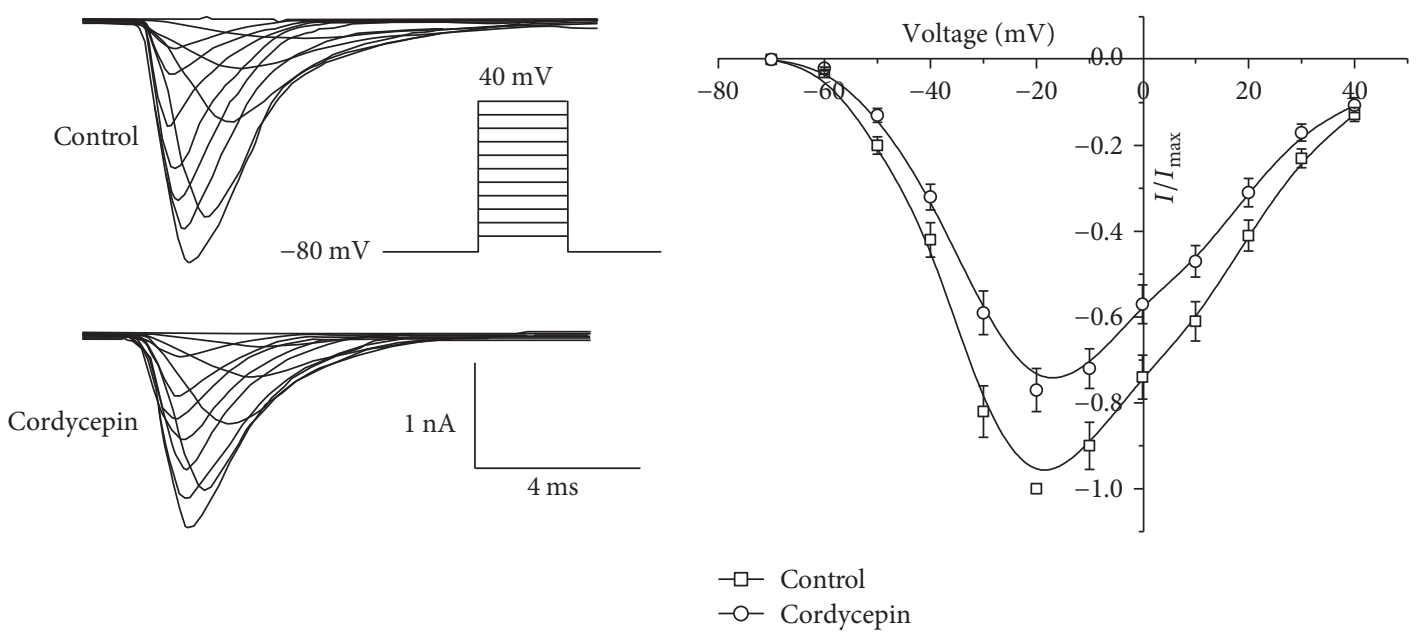

(a)

(b)

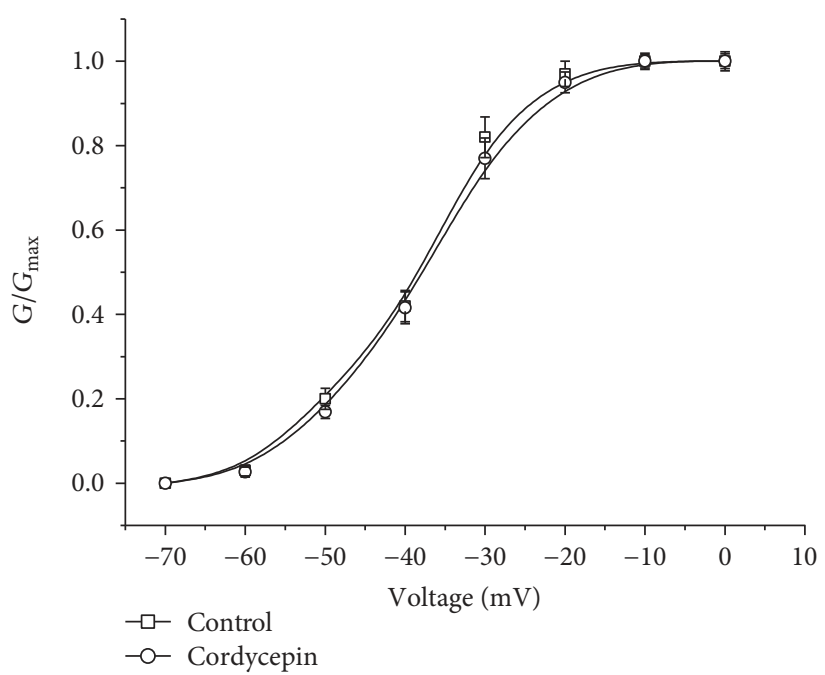

(c)

FIGURE 2: Effects of cordycepin on $I_{\mathrm{Na}}$ steady-state activation. (a) Representative traces of evoked $I_{\mathrm{Na}}$ before (top traces) and after (bottom traces) $80 \mu \mathrm{M}$ cordycepin application. (b) Current-voltage relationships of $I_{\mathrm{Na}}$ before and after $80 \mu \mathrm{M}$ cordycepin application. (c) Comparison of steady-state activation of $I_{\mathrm{Na}}$ before and after the $80 \mu \mathrm{M}$ cordycepin application.

3.3. Effect of Cordycepin on $I_{N a}$ Steady-State Inactivation. Figure 3(a) illustrates effects of cordycepin on voltage dependence of steady-state inactivation after examination with a dual-pulse protocol. Membrane potential was conditioned to different potentials (from $-100 \mathrm{mV}$ to $-10 \mathrm{mV}$, with $+10 \mathrm{mV}$ increment) for $50 \mathrm{~ms}$ and then depolarized to a fixed test potential of $-20 \mathrm{mV}$. Figure 3(a) displays representative $I_{\mathrm{Na}}$ traces before and after cordycepin treatment. Figure 3(b) presents comparison of inactivation curves before and after cordycepin treatment. The figure shows a significant shift in steady-state inactivation curves in the control and cordycepin treatment groups (control: $V_{1 / 2}=-47.4 \pm$ $3.7 \mathrm{mV}, n=10$; cordycepin: $-54.8 \pm 4.1, n=10 ; p<0.05)$. Application of cordycepin produced a $7.4 \mathrm{mV}$ negative shift in the inactivation curve.

3.4. Effect of Cordycepin on $I_{\mathrm{Na}}$ Recovery. Recovery time course of $I_{\mathrm{Na}}$ from inactivation was investigated using a dual-pulse protocol (Figure 4(a)). A conditioning step
( $50 \mathrm{~ms}$ ) from $-100 \mathrm{mV}$ to $-20 \mathrm{mV}$ was first employed to completely inactivate $I_{\mathrm{Na}}$. Then, after recovery at $-100 \mathrm{mV}$ for 1-20 ms, a test pulse of $-20 \mathrm{mV}$ was subsequently applied. Notably, after prolonged recovery (with recovery time from $1 \mathrm{~ms}$ to $20 \mathrm{~ms}$ ) at $-100 \mathrm{mV}$, the amplitude of $I_{\mathrm{Na}}$ gradually returned to control value (Figures 4(a) and 4(b)). Figure 4(b) presents comparison of percentages of peak current recovery from inactivation before and after cordycepin application. Recovery current amplitude (normalized with respect to precondition pulse induced the current amplitude) was plotted versus times before and after cordycepin application. Recovery time course from inactivation was well fitted by a single exponential function, with a recovery time constant of $1.48 \pm 0.06$ and $2.10 \pm 0.14 \mathrm{~ms}$ in the control $(n=10)$ and cordycepin $(n=10)(p<0.05)$ groups, respectively. Cordycepin significantly reduced the rate of $I_{\mathrm{Na}}$ recovery from inactivation. These results indicated that $I_{\mathrm{Na}}$ in the cordycepin-treated group recovered from inactivation more slowly than those in the control. 


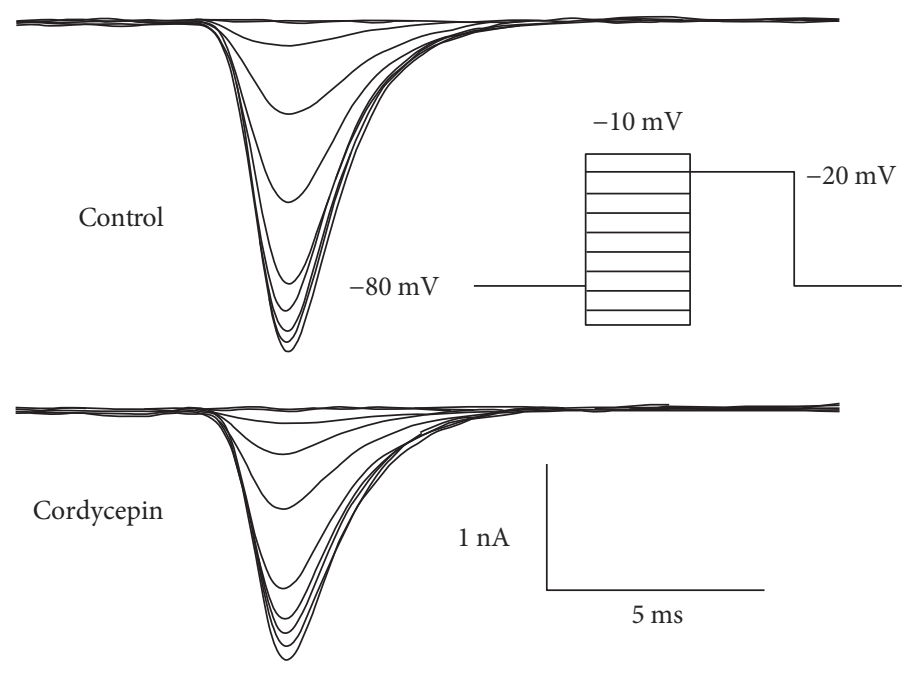

(a)

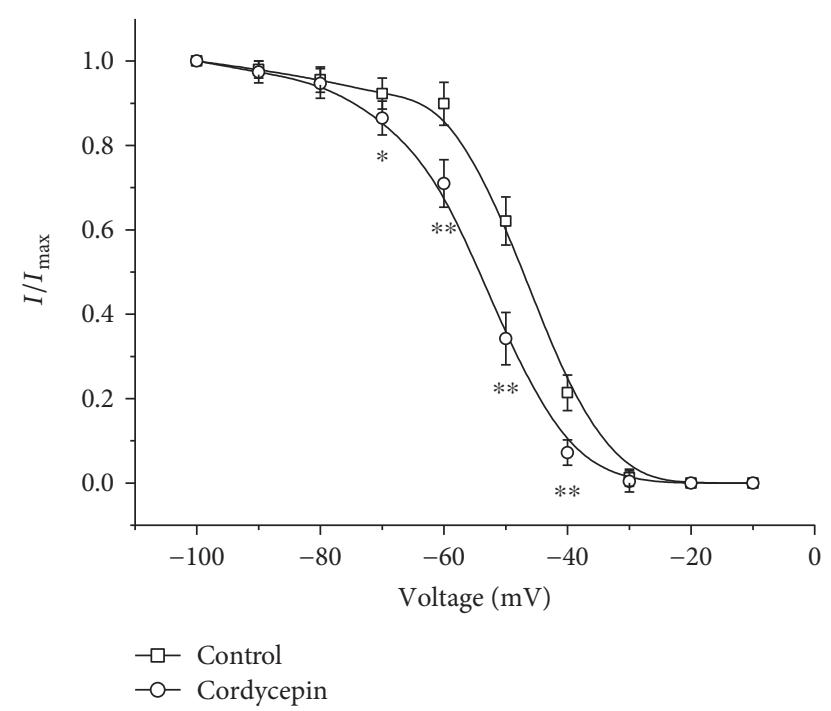

(b)

FIGURE 3: Effects of cordycepin on $I_{\mathrm{Na}}$ steady-state inactivation. (a) Current responses before (top traces) and after (bottom traces) $80 \mu \mathrm{M}$ cordycepin application examined with a dual-pulse protocols. (b) Comparison of steady-state inactivation of $I_{\mathrm{Na}}$ before and after the $80 \mu \mathrm{M}$ cordycepin application. ${ }^{*} p<0.05,{ }^{* *} p<0.01$ as compared with the control group.

3.5. Preapplication of Cordycepin-Induced Mild Inhibition on $I_{N a}$, Attenuating and Delaying the Subsequent HypoxiaInduced Rapid Dramatic Inhibition of $I_{N a}$. Studies demonstrated that hypoxia can induce rapid dramatic inhibition on $\mathrm{Na}^{+}$channels and current during initial stages $(1-3 \mathrm{~min})$ of hypoxia [3], which in turn resist the cell depolarization and reduce neuronal activity. This process will increase neuronal tolerance to low-oxygen environments $[3,23]$, indicating that there has been a self-adaptive cellular protective mechanism during initial stages of hypoxia. Thus, significant information can be obtained by investigating inhibition effects of cordycepin on $I_{\mathrm{Na}}$ during hypoxia, as account for neuroprotection effect of cordycepin from hypoxia insult $[13,15,16,24]$. Like the previous studies reported [3], there was a rapid dramatic inhibition of peak $I_{\mathrm{Na}}$ when the extracellular bath was changed from control perfusate to the hypoxic solution. After $3 \mathrm{~min}$ of hypoxic exposure, $I_{\mathrm{Na}}$ reduced to $50.6 \% \pm 5.12 \%$ of the baseline ( $n=12$; Figures $5(\mathrm{a})$ and $5(\mathrm{~b})$, Table $1 ; p<0.01)$. Steady-state inactivation was shifted by $-9.2 \pm 0.8 \mathrm{mV}$, and recovery time from inactivation also increased (recovery time constant in control: $1.51 \pm 0.06 \mathrm{~ms}$, $n=12$; hypoxia: $2.21 \pm 0.12 \mathrm{~ms}, n=12$; $p<0.05$ ). Interestingly, response of $I_{\mathrm{Na}}$ to hypoxia was markedly blocked with cordycepin after exposure to hypoxia for $3 \mathrm{~min}$ (Figures 5(a) and 5(b), Table 1) although mild inhibition on $I_{\mathrm{Na}}$ was observed after pretreatment with cordycepin (Figures 5(a) and 5(b), Table 1). When neurons were exposed to hypoxia for $3 \mathrm{~min}$ with cordycepin pretreatment, hypoxia-induced inhibition of $I_{\mathrm{Na}}$ was significantly attenuated $\left(66.3 \% \pm 5.53 \%\right.$ of initial $I_{\mathrm{Na}} ; n=12$; Figures $5(\mathrm{a})$ and $5(\mathrm{~b})$, Table 1$)$ compared with hypoxia only $(50.6 \% \pm 5.12 \%$ of initial $I_{\mathrm{Na}} ; n=12$; Figures 5(a) and 5(b), Table $\left.1 ; p<0.05\right)$. In the cordycepin pretreatment group, the descending slope $(4.6 \pm 0.32 \mathrm{mV} / \mathrm{min}, n=12$; Figure 5(b), Table 1) between 0 and 3 min after hypoxia treatment was obviously decreased when compared with hypoxia only $(15 \pm 0.11 \mathrm{mV} / \mathrm{min}, n=$ 12; Figure 5(b), Table 1). And most notably, the onset time of hypoxia-induced rapid dramatic inhibition on peak $I_{\mathrm{Na}}$ was also delayed from $0 \mathrm{~min}$ to $3 \mathrm{~min}$ in the cordycepin pretreatment group (Figure 5(b)), indicating that the neuron physical fitness response to external low-oxygen environments was improved through regulating self-adaptive cellular protective mechanism. No additive effects of hypoxia on the shift in steady-state inactivation and the time course of recovery from inactivation were observed (Table 1). These results indicated that mild inhibitory effect of cordycepin on $I_{\mathrm{Na}}$ channel may contribute to its neuroprotective effect against hypoxia insult.

\section{Discussion}

In the present study, we observed that cordycepin decreased the amplitude of $I_{\mathrm{Na}}$ in a concentration-dependent manner (Figure 1). Steady-state inactivation curves of $I_{\mathrm{Na}}$ shifted to more negative potentials (Figure 3), and time of $I_{\mathrm{Na}}$ recovery from inactivation was prolonged significantly by cordycepin (Figure 4). A negative shift on inactivation curve indicates low membrane potential threshold required for closing these channels. Slower recovery from inactivation implies prolonged transition of VGSC in cordycepin from inactivated to closed state and reduced fraction of available VGSC during spike trains [2]. These results imply that suppression of $I_{\mathrm{Na}}$ by cordycepin may inhibit intrinsic bursting and thus lead to a reduction in neuronal activity in CA1 neurons. This speculation was also confirmed by our previous study, which indicated that cordycepin can inhibit neuronal activity with low-frequency action potential bursting [17]. Furthermore, cordycepin pretreatment can significantly attenuate and delay hypoxia-induced rapid dramatic inhibition on $I_{\mathrm{Na}}$ (Figure 5, Table 1) with no additional effects on shifts in 

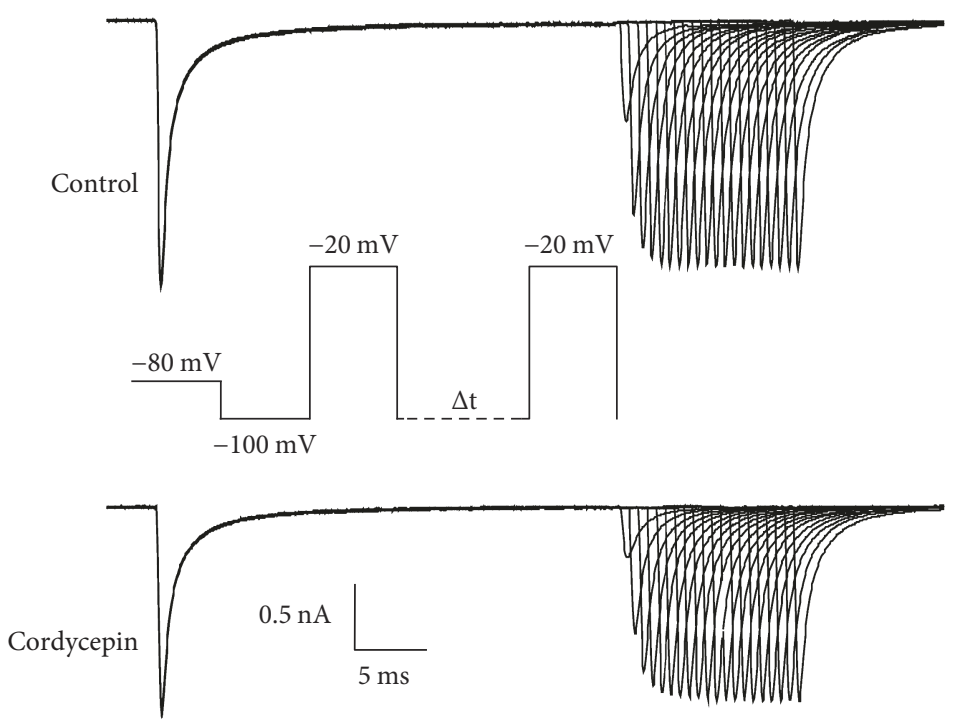

(a)

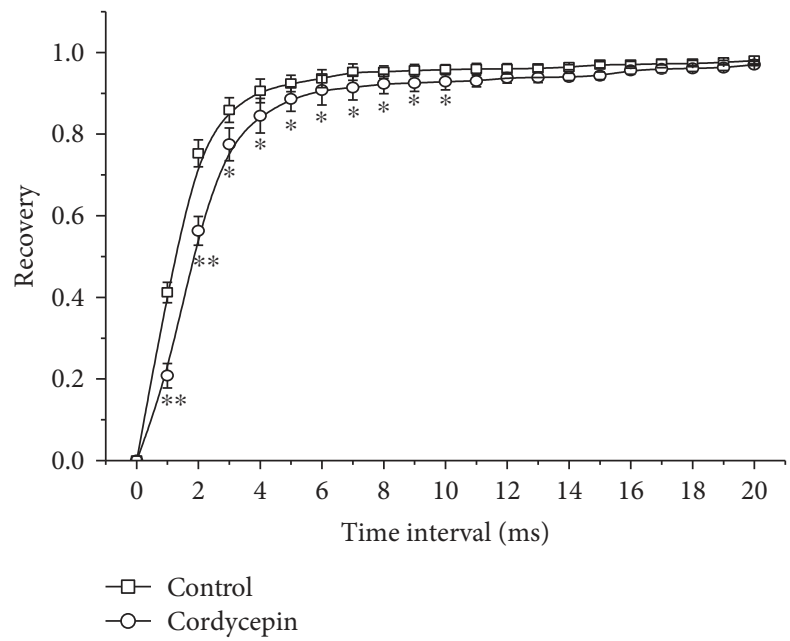

(b)

FIGURE 4: Effects of cordycepin on $I_{\mathrm{Na}}$ recovery from inactivation. (a) Representative traces of $I_{\mathrm{Na}}$ inactivation recovery before (top traces) and after (bottom traces) $80 \mu \mathrm{M}$ cordycepin application examined with a dual-pulse protocols. (b) Time courses of $I_{\mathrm{Na}}$ recovery from inactivation. ${ }^{*} p<0.05,{ }^{* *} p<0.01$ as compared with the control group.

steady-state inactivation and recovery time course from inactivation (Table 1). This result indicates that suppression effect of cordycepin on $I_{\mathrm{Na}}$ and $I_{\mathrm{Na}}$ kinetics may contribute to its neuroprotection from hypoxic insult.

$I_{\mathrm{Na}}$ is responsible for both action potential generation and propagation and therefore plays a crucial role in neuronal excitability $[1,2,25]$. Thus, $I_{\mathrm{Na}}$ modulation may possess biological significance. Previous studies suggested that influx of $\mathrm{Na}^{+}$contributes to brain damage during ischemia insult, as through activation of VGSC, $\mathrm{Na}^{+}$influx across neuronal membrane mediates sustained $\mathrm{Na}^{+}$entry, which in turn induces excessive membrane depolarization $[2-4,18,25]$. Consistently, evidence confirmed that excessive membrane depolarization may result from acute hypoxic or ischemic insults $[3,16,25,26]$. Hence, inhibition of $\mathrm{Na}^{+}$channel activation would reduce neuronal activity and reduce $\mathrm{Na}^{+}$ ion influx across neuronal membrane, which in turn against the hypoxia or ischemic induced the excessive membrane depolarization. Dong and $\mathrm{Xu}$ reported that mild inhibition in VGSC prolongs the duration, increases the threshold of excitation, and delays appearance of subsequent action potential, thus contributing to neuroprotection from hypoxic insult [2]. Other studies confirmed that reducing VGSC activity attenuates neuronal hypoxic responses and reduces hypoxia-induced neuronal injury and death in vitro and in vivo [2-5]. In the present study, we discovered that the application of cordycepin mildly inhibits VGSC (Figure 1), and it is coupled with a negative shift in steady-state inactivation (Figure 3) and slow time course of recovery from inactivation (Figure 4). Thus, we propose that cordycepin inhibition of VGSC may be an important mechanism to reduce neuronal activity, which in turn contributes to its neuroprotective effects against ischemic insults reported in our previous study $[16,17]$. 


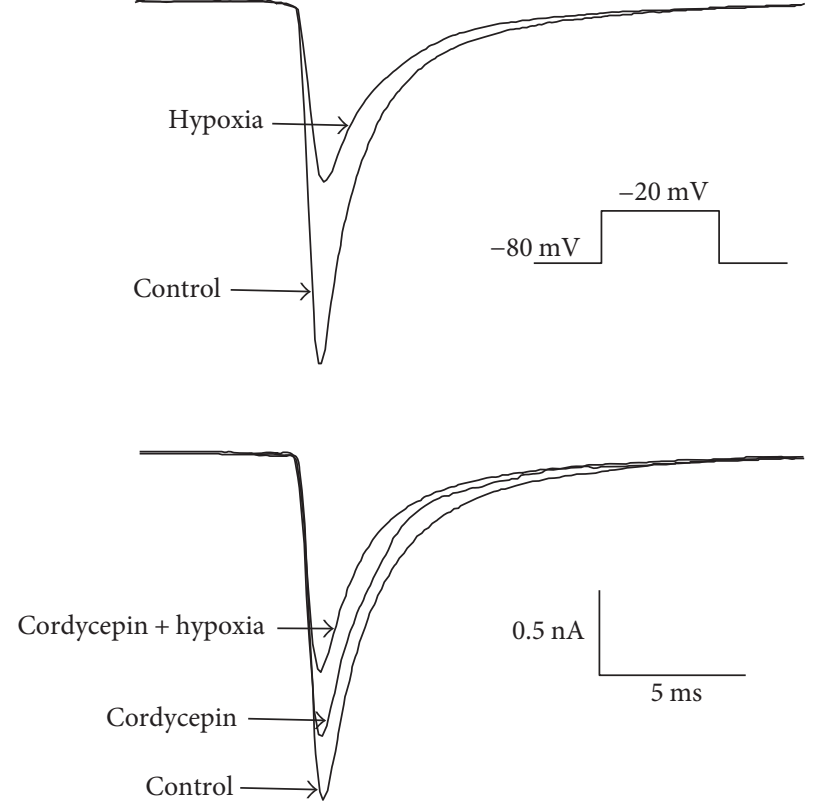

(a)

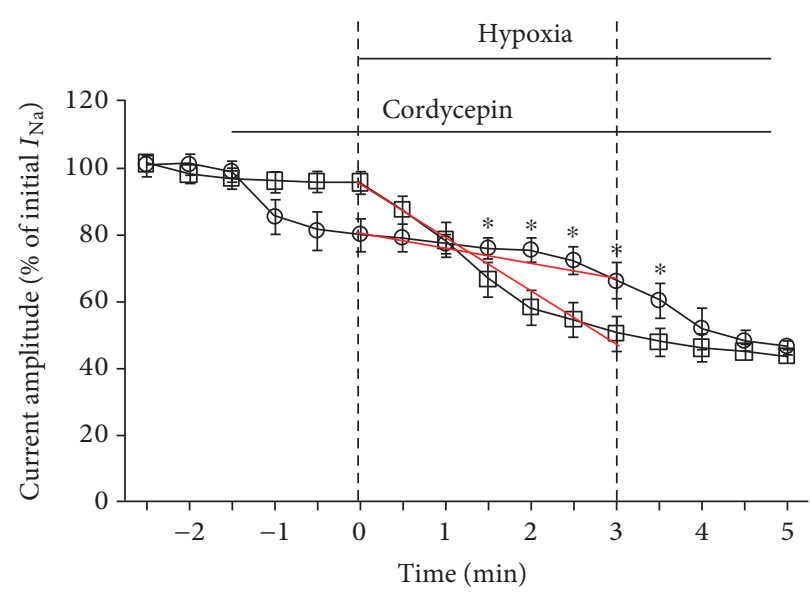

$\bigcirc$ Cordycepin + Hypoxia

$\square$ Hypoxia

FIgURE 5: Preapplication of cordycepin-induced mild inhibition on $I_{\mathrm{Na}}$ attenuated and delayed the subsequent hypoxia-induced rapid dramatic inhibition of $I_{\mathrm{Na}}$. (a) Hypoxia for $3 \mathrm{~min}$ induced dramatic inhibition on the traces of $I_{\mathrm{Na}}$ (top traces) in the absence of cordycepin. Reduction on $I_{\mathrm{Na}}$ induced by hypoxia was attenuated in the presence of cordycepin (bottom traces). (b) Comparison of suppression effects of hypoxia on $I_{\mathrm{Na}}$ in the absence and presence of cordycepin. The descending slope was indicated by the red line. Hypoxia-induced rapid dramatic reduction on $I_{\mathrm{Na}}$ was attenuated and delayed in the cordycepin pretreatment group. ${ }^{*} p<0.05$ as compared with the cordycepin pretreatment group.

TABle 1: Preapplication of cordycepin-induced mild inhibition on $I_{\mathrm{Na}}$ attenuated and delayed the subsequent hypoxia-induced rapid dramatic inhibition of $I_{\mathrm{Na}}$. (Recordings were collected after 3 min hypoxia.)

\begin{tabular}{|c|c|c|c|c|}
\hline Groups & $I_{\mathrm{Na}}$ amplitude (\% of initial) & Descending slope $(\mathrm{mV} / \mathrm{min})$ & Inactivation, $V_{1 / 2}(\mathrm{mV})$ & Recovery time (ms) \\
\hline Control $(n=10)$ & $96.8 \pm 3.8$ & & $-47.4 \pm 3.7$ & $1.48 \pm 0.06$ \\
\hline Cordycepin $(n=10)$ & $77.6 \pm 4.58^{\# \#}$ & & $-54.8 \pm 4.1^{\#}$ & $2.10 \pm 0.14^{\#}$ \\
\hline Hypoxia $(n=12)$ & $50.6 \pm 5.12^{\# \#}$ & $15 \pm 0.11$ & $-55.9 \pm 3.9^{\#}$ & $2.21 \pm 0.12^{\#}$ \\
\hline Cordycepin + hypoxia $(n=12)$ & $66.3 \pm 5.53^{*, \# \#}$ & $4.6 \pm 0.32^{* *}$ & $-54.3 \pm 3.8^{\#}$ & $2.26 \pm 0.14^{\#}$ \\
\hline
\end{tabular}

Descending slope means the inhibition rate induced by hypoxia from 0 to $3 \mathrm{~min} ;{ }^{*} p<0.05$ and ${ }^{* *} p<0.01$ compared to the hypoxia group; ${ }^{*} p<0.05$ and ${ }_{\# \#} p<0.01$ compared to the control group.

Additionally, to some extent, inhibition of $I_{\mathrm{Na}}$ is considered as a self-adaptive cellular protective mechanism during initial stages of hypoxia $[3-5,27]$. As inhibition of $\mathrm{Na}^{+}$channel activation reduces neuronal activity, this phenomenon results in reduction in energy demand at a time when energy production is severely compromised. This process ultimately increases neuronal tolerance to low-oxygen environments. Consistent with these deductions, we also noted that oxygen deprivation (hypoxia) causes rapid dramatic inhibition (within $3 \mathrm{~min}$ ) on the peak of $I_{\mathrm{Na}}$ with a negative shift in steady-state inactivation and prolonged recovery from inactivation (Figure 5). We also observed that cordycepin pretreatment can significantly attenuate and delay hypoxiainduced rapid dramatic inhibition on $I_{\mathrm{Na}}$ (Figure 5, Table 1) with no additive effects on the shift in steady-state inactivation and recovery time course from inactivation (Table 1). The descending slope was markedly decreased between 0 and $3 \mathrm{~min}$ hypoxia (Figure 5, Table 1 ), and the onset time of hypoxia-induced rapid dramatic inhibition on peak $I_{\mathrm{Na}}$ was delayed from $0 \mathrm{~min}$ to $3 \mathrm{~min}$ in the cordycepin pretreatment group (Figure 5(b)). These results demonstrate that preapplication of cordycepin-induced mild inhibition on $I_{\mathrm{Na}}$ attenuates and delays subsequent hypoxia-induced rapid dramatic inhibition of $I_{\mathrm{Na}}$, indicating that the neuron physical fitness response to external low-oxygen environments was improved through regulating self-adaptive cellular protective mechanism, which will ultimately increase neuronal tolerance to low-oxygen environments and thus save more rescue opportunities from further deterioration induced by hypoxia. However, further investigations are needed to clarify the underlying protective mechanism.

In conclusion, the present study revealed that cordycepin can reduce peak $I_{\mathrm{Na}}$ coupled with changes in voltage dependence of inactivation of $I_{\mathrm{Na}}$, and this mild reduction 
on $I_{\mathrm{Na}}$ attenuates and delays hypoxia-induced rapid dramatic decrease in the $I_{\mathrm{Na}}$. As modulation on $I_{\mathrm{Na}}$ in CA1 neurons occurs initially during ischemia, we propose that cordycepin-induced mild inhibition of $I_{\mathrm{Na}}$ is an important neuronal protective mechanism that may enhance neuronal tolerance to acute oxygen deprivation and delay hypoxia-induced neuronal injury.

\section{Conflicts of Interest}

The authors declare that there is no conflict of interests regarding the publication of this paper.

\section{Authors' Contributions}

Zhi-Bin Liu, Chao Liu, and Bin Zeng contributed equally to this study.

\section{Acknowledgments}

This work was supported by the National Natural Science Foundation of China (31660275, 81360205, 31171731, and 81660713), the Science and Technology Program of Education Department of Jiangxi Province (GJJ150792), Jiangxi Outstanding Youth Talent Cultivation Program (20171BCB23077), “555 talent project” of Jiangxi Province, and the Jiangxi Key Laboratory of Bioprocess Engineering and Co-Innovation Center for In Vitro Diagnostic Reagents and Devices of Jiangxi Province (20142BDH80003 and 2013-CXTD002).

\section{References}

[1] L. C. Kruger and L. L. Isom, "Voltage-gated $\mathrm{Na}^{+}$channels: not just for conduction," Cold Spring Harbor Perspectives in Biology, vol. 8, no. 6, article a029264, 2016.

[2] X. P. Dong and T. L. Xu, "Radix paeoniae rubra suppression of sodium current in acutely dissociated rat hippocampal CA1 neurons," Brain Research, vol. 940, no. 1-2, pp. 1-9, 2002.

[3] J. P. O’Reilly, T. R. Cummins, and G. G. Haddad, "Oxygen deprivation inhibits $\mathrm{Na}^{+}$current in rat hippocampal neurones via protein kinase C," The Journal of Physiology, vol. 503, Part 3, pp. 479-488, 1997.

[4] R. J. Docherty and C. E. Farmer, "The pharmacology of voltage-gated sodium channels in sensory neurones," Handbook of Experimental Pharmacology, vol. 194, pp. 519-561, 2009.

[5] V. Zuliani, A. Rapalli, M. K. Patel, and M. Rivara, "Sodium channel blockers: a patent review (2010 - 2014)," Expert Opinion on Therapeutic Patents, vol. 25, no. 3, pp. 279-290, 2015.

[6] H. S. Tuli, A. K. Sharma, S. S. Sandhu, and D. Kashyap, "Cordycepin: a bioactive metabolite with therapeutic potential," Life Sciences, vol. 93, no. 23, pp. 863-869, 2013.

[7] P. K. Wu, Z. Tao, Z. Ouyang et al., "The anti-tumor effects of cordycepin-loaded liposomes on the growth of hepatoma 22 tumors in mice and human hepatoma BEL-7402 cells in culture," Drug Development and Industrial Pharmacy, vol. 42, no. 9, pp. 1424-1433, 2016.

[8] X. Tian, Y. Li, Y. Shen, Q. Li, Q. Wang, and L. Feng, “Apoptosis and inhibition of proliferation of cancer cells induced by cordycepin (review)," Oncology Letters, vol. 10, no. 2, pp. 595-599, 2015.

[9] J. Y. Yoon, J. H. Kim, K. S. Baek et al., “A direct protein kinase B-targeted anti inflammatory activity of cordycepin from artificially cultured fruit body of Cordyceps militaris," Pharmacognosy Magazine, vol. 11, no. 43, pp. 477-485, 2015.

[10] Y. H. Choi, G. Y. Kim, and H. H. Lee, "Anti-inflammatory effects of cordycepin in lipopolysaccharide-stimulated RAW 264.7 macrophages through Toll-like receptor 4-mediated suppression of mitogen-activated protein kinases and NF- $\kappa \mathrm{B}$ signaling pathways," Drug Design, Development and Therapy, vol. 8, pp. 1941-1953, 2014.

[11] S. Shin, S. Lee, J. Kwon et al., "Cordycepin suppresses expression of diabetes regulating genes by inhibition of lipopolysaccharide-induced inflammation in macrophages," Immune Network, vol. 8, no. 3, pp. 98-105, 2009.

[12] L. Ma, S. Zhang, and M. Du, "Cordycepin from Cordyceps militaris prevents hyperglycemia in alloxan-induced diabetic mice," Nutrition Research, vol. 35, no. 5, pp. 431-439, 2015.

[13] Z. Cheng, W. He, X. Zhou et al., "Cordycepin protects against cerebral ischemia/reperfusion injury in vivo and in vitro," European Journal of Pharmacology, vol. 664, no. 1-3, pp. 20 28, 2011.

[14] C. Dou, Z. Cao, N. Ding et al., "Cordycepin prevents bone loss through inhibiting osteoclastogenesis by scavenging ROS generation," Nutrients, vol. 8, no. 4, p. 231, 2016.

[15] I. K. Hwang, S. S. Lim, K. Y. Yoo et al., "A phytochemically characterized extract of Cordyceps militaris and cordycepin protect hippocampal neurons from ischemic injury in gerbils," Planta Medica, vol. 74, no. 2, pp. 114-119, 2008.

[16] C. Chen, X. P. Liu, W. Jiang et al., "Anti-effects of cordycepin to hypoxia-induced membrane depolarization on hippocampal CA1 pyramidal neuron," European Journal of Pharmacology, vol. 796, pp. 1-6, 2017.

[17] L. H. Yao, C. H. Li, W. W. Yan, J. N. Huang, W. X. Liu, and P. Xiao, "Cordycepin decreases activity of hippocampal CA1 pyramidal neuron through membrane hyperpolarization," Neuroscience Letters, vol. 503, no. 3, pp. 256-260, 2011.

[18] Q. Mao, F. Jia, X. H. Zhang et al., "The up-regulation of voltage-gated sodium channel Nav1.6 expression following fluid percussion traumatic brain injury in rats," Neurosurgery, vol. 66, no. 6, pp. 1134-1139, 2010.

[19] L. H. Yao, L. P. Huang, Q. P. Xiong et al., "Modulation effects of cordycepin on voltage-gated cation channels on hippocampal CA1 pyramidal neuron," Latin American Journal of Pharmacy, vol. 33, no. 6, pp. 954-959, 2014.

[20] J. Wang, J. E. Cottrell, and I. S. Kass, "Effects of desflurane and propofol on electrophysiological parameters during and recovery after hypoxia in rat hippocampal slice CA1 pyramidal cells," Neuroscience, vol. 160, no. 1, pp. 140-148, 2009.

[21] H. Ni, X. H. Zhou, H. H. Li, and W. F. Huang, "Column chromatographic extraction and preparation of cordycepin from Cordyceps militaris waster medium," Journal of Chromatography B, vol. 877, no. 22, pp. 2135-2141, 2009.

[22] L. H. Yao, J. N. Huang, C. H. Li et al., "Cordycepin suppresses excitatory synaptic transmission in rat hippocampal slices via a presynaptic mechanism," CNS Neuroscience \& Therapeutics, vol. 19, no. 4, pp. 216-221, 2013.

[23] C. C. Cocilova and S. L. Milton, "Characterization of brevetoxin $(\mathrm{PbTx}-3)$ exposure in neurons of the anoxia-tolerant 
freshwater turtle (Trachemys scripta)," Aquatic Toxicology, vol. 180, pp. 115-122, 2016.

[24] S. H. Lee, I. G. Ko, S. E. Kim et al., "Aqueous extract of Cordyceps alleviates cerebral ischemia-induced short-term memory impairment in gerbils," Journal of Exercise Rehabilitation, vol. 12, no. 2, pp. 69-78, 2016.

[25] X. X. Dong, Y. Wang, and Z. H. Qin, "Molecular mechanisms of excitotoxicity and their relevance to pathogenesis of neurodegenerative diseases," Acta Pharmacologica Sinica, vol. 30, no. 4, pp. 379-387, 2009.

[26] H. S. Sun, Z. P. Feng, T. Miki, S. Seino, and R. J. French, "Enhanced neuronal damage after ischemic insults in mice lacking Kir6.2-containing ATP-sensitive $\mathrm{K}^{+}$channels," Journal of Physiology, vol. 95, no. 4, pp. 2590-2601, 2006.

[27] P. Calabresi, A. Pisani, N. B. Mercuri, and G. Bernardi, "On the mechanisms underlying hypoxia-induced membrane depolarization in striatal neurons," Brain, vol. 118, Part 4, pp. 1027-1038, 1995. 

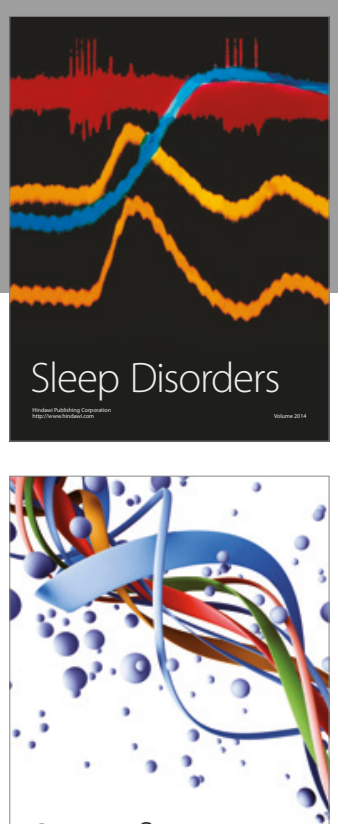

Scientifica
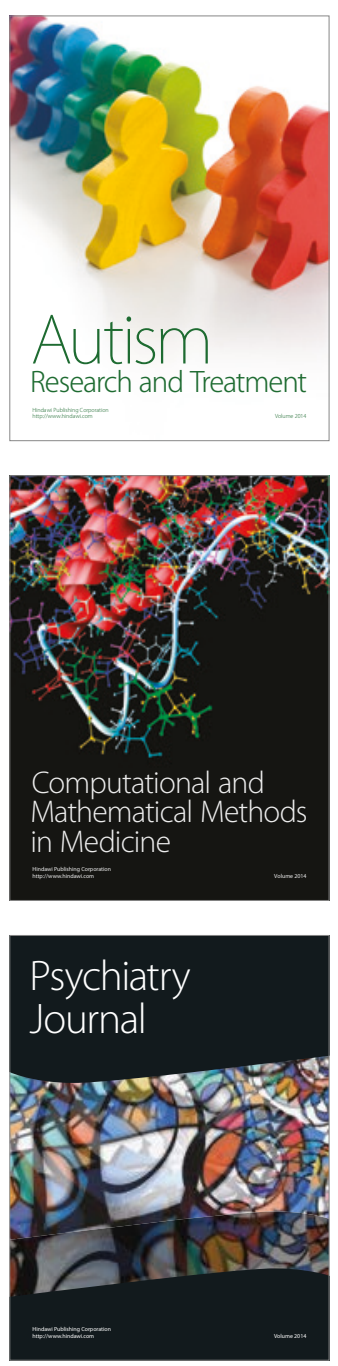
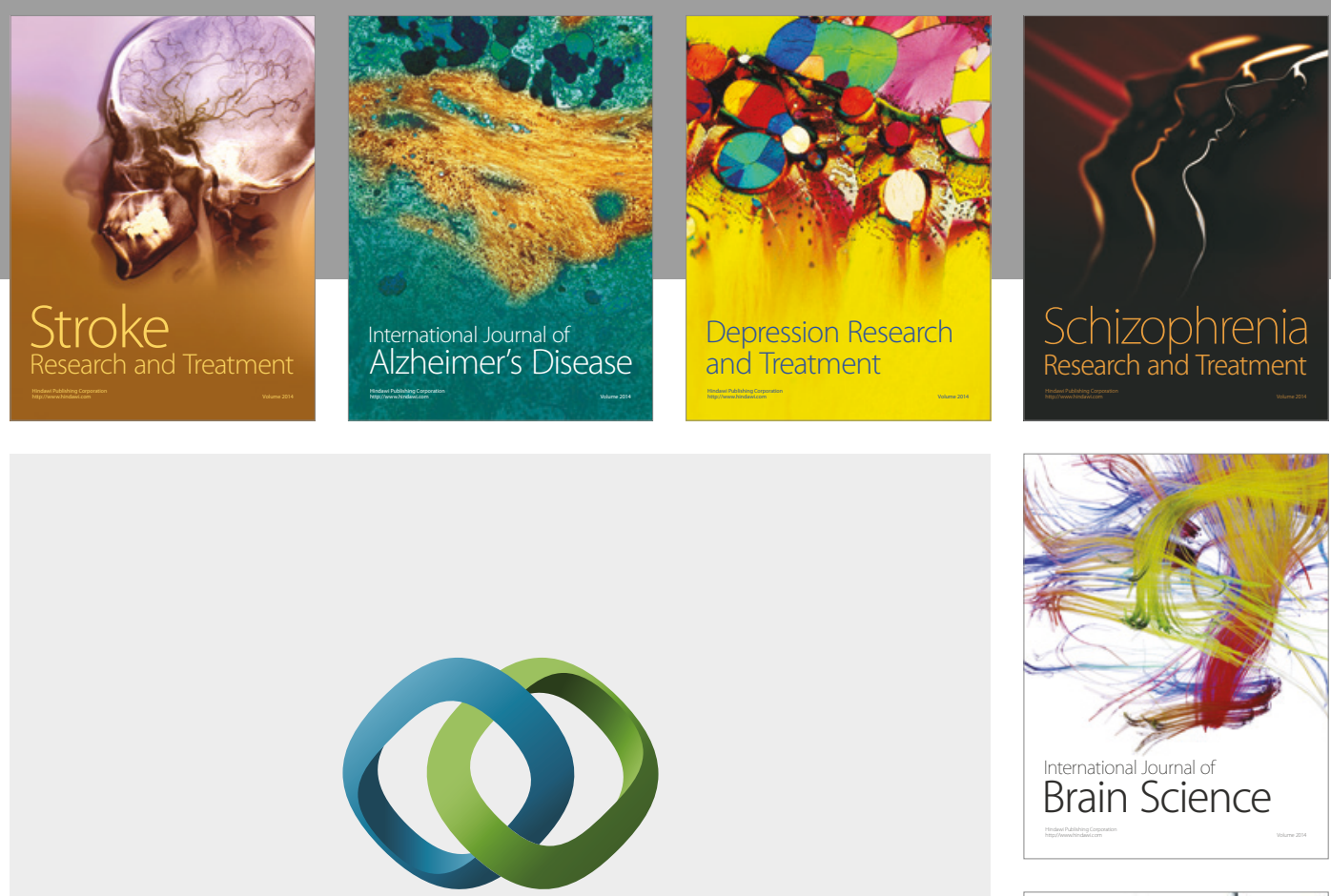

\section{Hindawi}

Submit your manuscripts at

https://www.hindawi.com
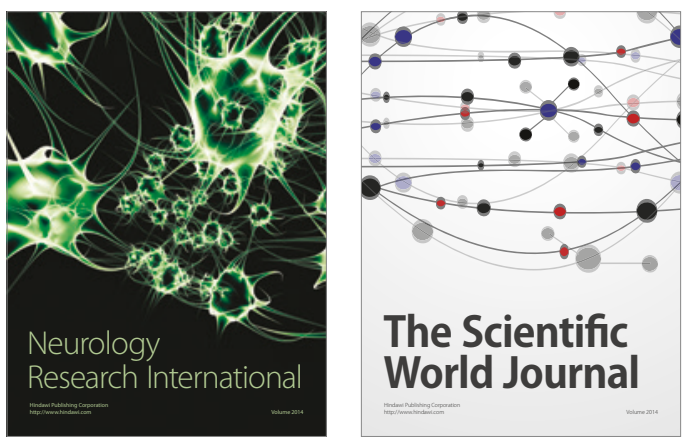

The Scientific World Journal

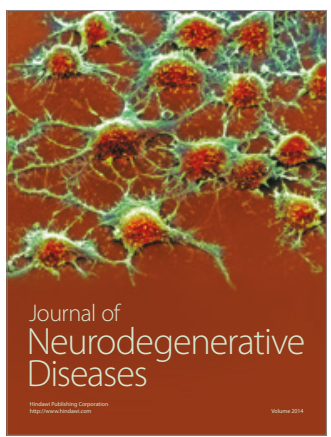

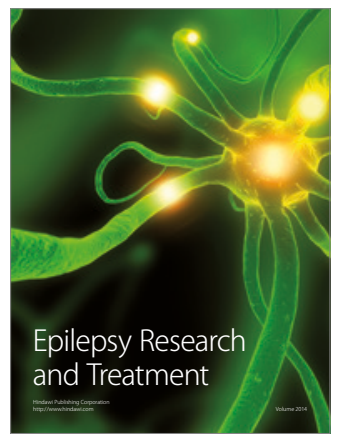

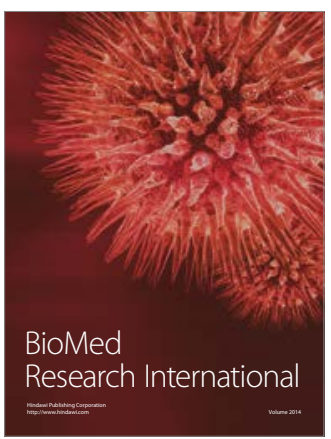

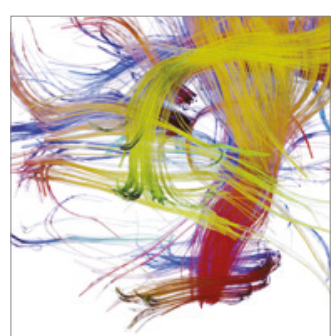

Brain Science

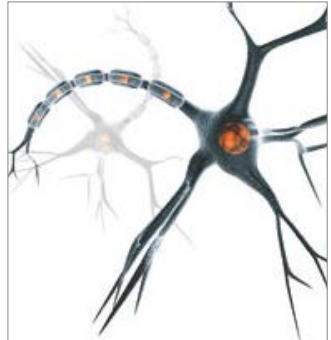

Neural Plasticity
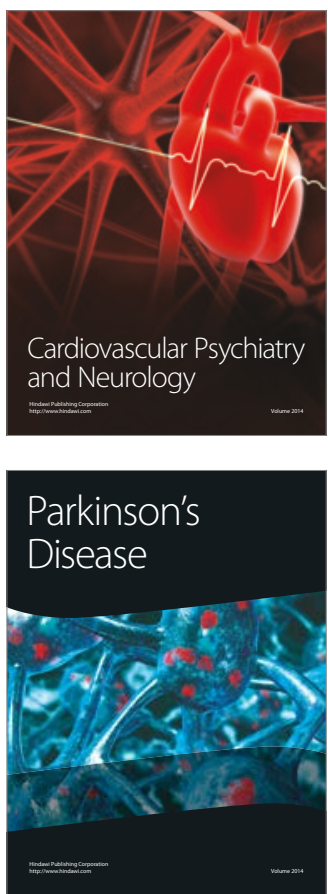